\title{
EDM Experiments at Storage Rings
}

\author{
Hans Ströher ${ }^{1 *}$ \\ ${ }^{1}$ Institut für Kernphysik, Forschungszentrum Jülich, 52425 Jülich, Germany
}

\begin{abstract}
According to our present understanding, the early Universe contained the same amount of matter and anti-matter and, if the Universe had behaved symmetrically as it developed, every particle would have been annihilated by one of its antiparticles. One of the great mysteries in the natural sciences is therefore, why matter dominates over antimatter in the visible Universe. The breaking of the combined chargeconjugation and parity symmetries (CP-violation, CPV) in the Standard Model of particle physics (SM) is insufficient to explain this and further sources of CPV must be sought. These could manifest themselves in electric dipole moments (EDMs) of elementary particles, which occur when the centroids of positive and negative charges are mutually and permanently displaced. An EDM observation would also be an indication for physics beyond the SM. No EDM has been observed so far; after its discovery, investigations on different systems will be required to pin down CPV sources. In this respect the idea to search for EDMs of charged hadrons (i.p. proton and deuteron) in a new class of precision storage rings has recently been put forward. In addition, such searches bear the potential to reach sensitivities of $10^{-29} \mathrm{e} \cdot \mathrm{cm}$. The EDM measurement principle, the time development of the polarization vector subject to a perpendicular electric field, is simple, but the smallness of the effect makes this an enormously challenging project. A stepwise approach, from R\&D for key-technologies towards the holy grail of a double-beam precision storage ring with counter-rotating beams, is needed. The ongoing research of the JEDI Collaboration at the cooler synchrotron COSY of Forschungszentrum Jülich (Germany) provides invaluable information: recent experimental milestones are presented and discussed.
\end{abstract}

\section{Introduction}

A new class of precision storage rings is required to search for electric dipole moments (EDMs) of charged particles with unprecedented sensitivity. In recent years, first decisive steps towards the design and construction of such a facility have been taken by establishing the required key technologies. One future milestone is to deliver the first directly measured EDMs for both the proton and the deuteron using the existing cooler storage ring COSY at Forschungszentrum Jülich (FZJ) in Jülich (Germany), thus providing the proof-of-principle for this method.

\section{Background}

Permanent EDMs of particles violate both time reversal (T) and parity (P) invariance and, on the basis of the CPT theorem, they also violate the combined symmetry $\mathrm{CP}$ (CPV). Such a symmetry breaking is thought to be responsible for the different behaviour of particles and antiparticles, leading, e.g., to the apparent matterantimatter asymmetry in the Universe. CPV is found in the electroweak part of the Standard Model of particle physics (SM) but, since SM-CPV is much too weak to explain the matter-antimatter asymmetry, other sources must be sought. An obvious candidate to investigate is an EDM of a fundamental particle: no EDM has yet been observed, but they must exist. Finding a finite EDM would very probably also indicate new physics, i.e., interactions not contained in the SM. Even after a possible EDM observation, different systems would have to be investigated in order to identify the CPV-source. EDMs are very small - the best current limit for the neutron is $3 \times 10^{-26} \mathrm{e} \cdot \mathrm{cm}[1]$; in addition to free particles, there are searches in atoms and molecules that have reached even smaller upper limits - and the aim for charged particles is at least $10^{-29} \mathrm{e} \cdot \mathrm{cm}$. Although the measurement principle - the time development of the polarization vector subject to a perpendicular electric field - is simple, this represents an enormously challenging project due to the smallness of the expected effect.

It should be noted that, whereas charged particles potentially offer the highest sensitivity with discovery potential, up to now no EDM measurements for protons and deuterons in a storage ring have been performed. However, an upper limit of $10^{-19} \mathrm{e} \cdot \mathrm{cm}$ was obtained for muons as a side activity of the measurement of the anomalous magnetic moment $\left((\mathrm{g}-2)_{\mu}\right)$ in a storage ring experiment.

Given the history of neutron EDM searches over the past 50 years or so, it is evident that a corresponding level of

\footnotetext{
Corresponding author: h.stroeher@fz-juelich.de
} 
sensitivity cannot be obtained in one giant leap - a stepwise approach, in which the next generation measurement is based on the expertise and technological know-how gained in the preceding one, is compulsory. The steps towards the holy grail of a charged particle EDM search, using a double-beam precision storage ring with counter-rotating beams, comprise:

1. Research and development of all the key techniques at an existing conventional single-beam storage ring. COSY, the cooler synchrotron and storage ring at the Forschungszentrum Jülich, is the ideal place for these investigations, which involve spin-coherence time optimization, precision polarimetry development, beam tracking measurements, etc.;

2. Precision spin-tracking simulations: an essential requirement for an assessment of the capabilities of the final precision ring is to provide realistic simulations, e.g., for benchmarking EDM test installations in COSY;

3. A proof-of-principle experiment: this measurement will use COSY-Jülich without major transformations except for improvements of the beam position monitor (BPM) system and the assembly of a radiofrequency (RF) Wien-filter which (in an ideal storage ring) would induce spin rotation due to an EDM;

4. A first direct EDM measurement (for the proton and the deuteron), again exploiting COSY-Jülich, but here upgraded with a short electrostatic deflector inside the ring. The beam chicane could be inserted in one of the straight sections of COSY. This would test one of the key techniques of the final ring and should lead to an EDM upper limit in the order of $10^{-24} \mathrm{e} \cdot \mathrm{cm}$. It would also provide a reality check on further key items, e.g., the spin coherence time and polarimetry;

5. Conceptual design report (CDR) and technical design report (TDR) for the final dedicated storage ring, including cost estimates for building and operating the facility;

6. Construction and commissioning of the new facility for EDM searches, once funding has been secured.

In the following, recent achievements for step 1., obtained by the JEDI (Jülich Electric Dipole moment Investigations) collaboration [2] at COSY-Jülich, are presented. It should be mentioned that this research is supported by an Advanced Grant of the European Research Council (ERC AdG "srEDM") [3], which the reader is referred to for further information.

\section{Experimental achievements}

\subsection{Ultra-high precision spin tune measurement}

A new method to determine the spin tune was established and tested [4]. In an ideal planar magnetic storage ring, the spin tune - defined as the number of spin precessions per turn - is given by $v_{\mathrm{s}}=\gamma \mathrm{G}(\gamma$ is the
Lorentz factor, $\mathrm{G}$ the gyromagnetic anomaly). At 970 $\mathrm{MeV} / \mathrm{c}$, the deuteron spins coherently precess at a frequency of about $120 \mathrm{kHz}$ in COSY. The spin tune was deduced from the up-down asymmetry of deuteroncarbon scattering. In a time interval of $2.6 \mathrm{~s}$, the spin tune was determined with a precision of the order $10^{-8}$, and to $10^{-10}$ for a continuous $100 \mathrm{~s}$ accelerator cycle. This renders the new method a precision tool for accelerator physics; observing and controlling the spin motion of particles to high precision is mandatory, in particular, for the measurement of electric dipole moments of charged particles in a storage ring.

\subsection{Record in-plane polarization lifetime}

A deuteron beam polarization lifetime near 1000 seconds in the horizontal plane of the magnetic storage ring COSY has been observed [5]. This long spin coherence time was maintained through a combination of beam bunching, electron cooling, sextupole field corrections, and the suppression of collective effects through beam current limits. This record lifetime is required for a storage ring search for an intrinsic electric dipole moment on the deuteron at a statistical sensitivity level approaching $10^{-29} \mathrm{e} \cdot \mathrm{cm}$.

\subsection{Feedback system for an active control of the spin tune}

The successful use of feedback from a spin polarization measurement to the revolution frequency of a 0.97 $\mathrm{GeV} / \mathrm{c}$ bunched and polarized deuteron beam in the Cooler Synchrotron (COSY) storage ring has been realized in order to control both the precession rate $(\approx$ $120 \mathrm{kHz}$ ) and the phase of the horizontal polarization component [6]. Real time synchronization with a radio frequency (RF) solenoid made possible the rotation of the polarization out of the horizontal plane, yielding a demonstration of the feedback method to manipulate the polarization. In particular, the rotation rate shows a sinusoidal function of the horizontal polarization phase (relative to the RF solenoid), controlled to within a one standard deviation range of $\sigma=0.21 \mathrm{rad}$. The minimum possible adjustment was $3.7 \mathrm{mHz}$ out of a revolution frequency of $753 \mathrm{kHz}$, which changes the precession rate by $26 \mathrm{mrad} / \mathrm{s}$. Such capability meets the requirement for the use of storage rings to look for an intrinsic electric dipole moment of charged particles.

\subsection{Spin tune mapping as a novel tool to probe spin dynamics in storage rings}

An extended paper entitled "Spin tune mapping as a novel tool to probe the spin dynamics in storage rings" has recently been published [7]. It was motivated by the fact that precision experiments, such as the search for electric dipole moments of charged particles using storage rings, demand understanding of the spin dynamics with unprecedented accuracy. A new method 
artificially applied longitudinal magnetic fields, called "spin tune mapping", has been developed. The technique was experimentally tested in 2014 at COSY and, for the first time, the angular orientation of the stable spin axis at two different locations in the ring has been determined to an unprecedented accuracy of better than $2.8 \mu \mathrm{rad}$.

\section{Data base experiment}

For the deuteron EDM search, a polarimeter (reaction) is required that maximizes efficiency and sensitivity to the deuteron vector polarized beam in the storage ring. Such a polarimeter is likely to be based on a thick carbon target, because the spin-orbit effects in elastic scattering are large. The data required to optimize the polarimeter design have been taken with the refurbished forward part of the WASA-at-COSY detector [8] for a variety of deuteron energies and carbon as well as $\mathrm{CH}_{2}$ ribbon targets. Analysis of the data has started - once completed its results will be used in simulations of various polarimeter designs.

\section{Technical developments}

\subsection{RF Wien filter}

A novel waveguide RF Wien filter [9] is required for the proof-of-principle EDM measurement (the so called precursor experiment; see below) in a conventional storage ring like COSY. By design the orthogonality of the electric and magnetic field is accomplished. In order to maintain an optimal Lorentz-force compensation, the beam width must be kept as small as possible. Inside COSY this is achieved at the so called PAX low- $\beta$ section [10].

\subsection{Electrostatic deflector}

For the final EDM ring, high-field electrostatic deflectors have to be developed. The deflector development for a final deuteron EDM ring, which requires combined $E x B$ deflectors, is focused on highfield devices of roughly $17 \mathrm{MV} / \mathrm{m}$. Such high electrostatic field gradients could already be achieved on a test bench at RWTH Aachen University [11]. In addition, a first technical design for an electromagnetic deflector was completed and a prototype test device will be produced to further investigate and optimize the field gradient of high electrostatic fields in superimposed magnetic fields.

\subsection{EDM polarimeter}

A new polarimeter, based on LYSO scintillator modules with SiPM readout, is under development at our institute [12]. Test with extracted deuteron beams from COSY setup. The complete system, currently comprising 52 modules, is planned to supersede the currently used COSY polarimeters (EDDA, WASA) in the near future.

\section{Planned precursor experiment}

For 2018, the JEDI collaboration plans a first measurement of the deuteron EDM at COSY. In a pure magnetic storage ring such as COSY, an EDM will generate an oscillation of the vertical polarization component. For a $970 \mathrm{MeV} / \mathrm{c}$ deuteron beam with the spin precession frequency of $120 \mathrm{kHz}$, a tiny amplitude is expected, e.g., $3 \cdot 10^{-10}$ for an EDM of $\mathrm{d}=10^{-24} \mathrm{e} \cdot \mathrm{cm}$. To allow for a build-up of the vertical polarization proportional to the EDM, a so called radio-frequency (RF) Wien-filter has to be operated [8]. A prototype was successfully installed and operated in COSY in 2014. A new device with a stronger magnetic field $(0.05 \mathrm{~T} \cdot \mathrm{mm})$ was developed and constructed together with the Institut für Hochfrequenztechnik (IHF) at RWTH Aachen University and ZEA-1 in Jülich. This new RF Wienfilter was installed in COSY in May 2017. A first commissioning run was successfully conducted in June 2017.

\section{Acknowledgements}

The author wishes to acknowledge the support by the European Research Council via the ERC AdG "srEDM" (\#694340). He also thanks all involved members of the JEDI collaboration and of the Institut für Kernphysik of Forschungszentrum Jülich for their dedication and persistence towards this long-term project.

\section{References}

1. J. M. Pendlebury et al. Phys. Rev. D 92, 092003 (2015)

2. JEDI collaboration at COSY-Jülich; see: http://collaborations.fz-juelich.de/ikp/jedi/

3. H. Ströher (PI): ERC AdG srEDM (\#694340); see: http://sredm-ercgrant.de/

4. D. Eversmann et al. (JEDI Collaboration) Phys. Rev. Lett. 115, 094801 (2015)

5. G. Guidoboni et al. (JEDI Collaboration) Phys. Rev. Lett. 117, 054801 (2016)

6. N. Hempelmann et al. (JEDI Collaboration) Phys. Rev. Lett. 119, 014801 (2017)

7. A. Saleev et al. (JEDI Collaboration) Phys. Rev. Accel. Beams 20, 072801 (2017)

8. WASA-at-COSY; see: http://collaborations.fz-juelich.de/ikp/wasa/

9. J. Slim et al., Nucl. Instr. Meth. A 828, 116 (2016); J. Slim et al., Nucl. Instr. Meth. A 859, 52 (2017) 
10. C. Weidemann et al., Phys. Rev. ST-AB 18, 020101 (2015)

11. Institut für Kernphysik (IKP) Annual Report 2015 and 2016, Berichte des Forschungszentrums Jülich: Jül-4393, Jül-4398

see: http://www.fz-juelich.de/ikp/EN/Service/

12. JEDI contributions to CALOR2016 (accessible via Ref. [2] above) 